\title{
The Holographic Supercurrent Anomaly
}

\author{
M. Chaichian ${ }^{\dagger}$ and W.F. Chen ${ }^{\ddagger}$ \\ High Energy Physics Division, Department of Physical Sciences, University of Helsinki \\ and \\ Helsinki Institute of Physics, FIN-00014, Helsinki, Finland
}

\begin{abstract}
The $\gamma$-trace anomaly of supersymmetry current in a supersymmetric gauge theory shares a superconformal anomaly multiplet with the chiral $R$-symmetry anomaly and the Weyl anomaly, and its holographic reproduction is a valuable test to the AdS/CFT correspondence conjecture. We investigate how the $\gamma$-trace anomaly of the supersymmetry current of $\mathcal{N}=1$ four-dimensional supersymmetric gauge theory in an $\mathcal{N}=1$ conformal supergravity background can be extracted out from the $\mathcal{N}=2$ gauged supergravity in five dimensions. It is shown that the reproduction of this super-Weyl anomaly originates from the following two facts: First the $\mathcal{N}=2$ bulk supersymmetry transformation converts into $\mathcal{N}=1$ superconformal transformation on the boundary, which consists of $\mathcal{N}=1$ supersymmetry transformation and special conformal supersymmetry (or super-Weyl) transformation; second the supersymmetry variation of the bulk action of five-dimensional gauged supergravity is a total derivative. The non-compatibility of supersymmetry and the superWeyl transformation invariance yields the holographic supersymmetry current anomaly. Furthermore, we speculate on that the contribution from the external gauge and gravitational background fields to the superconformal anomaly may have different holographic origin.
\end{abstract}

PACS: 04.65.+e, 11.30.Pb, 11.15.-q, 11.40.-q

Keywords: AdS/CFT correspondence; Superconformal anomaly; Gauged supergravity; Holography; Supersymmetry; Brane dynamics

\section{INTRODUCTION}

The AdS/CFT correspondence conjecture [1] states that the type IIB string theory compactified on $A d S_{5} \times S^{5}$ theory with $N$ units of $R-R$ flux on $S^{5}$ describes the same physics

\footnotetext{
*E-mail: Masud.Chaichian@helsinki.fi

${ }^{\dagger}$ E-mail: Wen-feng.Chen@helsinki.fi
} 
as $\mathcal{N}=4 S U(N)$ supersymmetric Yang-Mills theory. Further, the explicit definition was given in Refs. [2] and [3] as the following. Given the type IIB superstring theory in the background $A d S_{d+1} \times X^{9-d}$, with $X^{9-d}$ being a compact Einstein manifold, the boundary effect of the type IIB superstring theory must be considered since $A d S_{d+1}$ has a boundary at spatial infinity, which is actually a copy of Minkowski space $M_{d}$ (or its compactified version $\left.S_{d}\right)$. The partition function of such a string theory with certain boundary value of a bulk field takes the following form,

$$
\left.Z_{\text {String }}[\phi]\right|_{\phi \rightarrow \phi_{0}}=\int_{\phi(x, 0)=\phi_{0}(x)} \mathcal{D} \phi(x, r) \exp (-S[\phi(x, r)]),
$$

where $\phi^{(0)}(x)$ is the boundary value of the $A d S_{d+1}$ bulk quantity $\phi(x, r)$ such as the graviton, gravitino, $N S-N S$ and $R-R$ antisymmetric tensor fields etc.. Since the isometry group $S O(2, d)$ of $A d S_{d+1}$ acts as the conformal group on $M_{d}$, and hence a quantum field theory defined on the boundary should be conformal invariant. The $A d S / C F T$ correspondence conjecture means that the type IIB superstring partition function (1) should be identical to the generating functional for the correlation functions of the composite operators of certain conformal field theory

$$
\begin{aligned}
Z_{\mathrm{CFT}}\left[\phi_{0}\right] & =\left\langle\exp \int_{M^{d}} d^{d} x \mathcal{O}(x) \phi_{0}(x)\right\rangle \\
& =\sum_{n} \frac{1}{n !} \int \prod_{i=1}^{n} d^{d} x_{i}\left\langle\mathcal{O}_{1}\left(x_{1}\right) \cdots \mathcal{O}_{n}\left(x_{n}\right)\right\rangle \phi_{0}\left(x_{1}\right) \cdots \phi_{0}\left(x_{n}\right) \\
& \equiv \exp \left(-\Gamma_{\mathrm{CFT}}\left[\phi_{0}\right]\right),
\end{aligned}
$$

$\Gamma\left[\phi_{0}\right]$ being the quantum effective action describing the composite operators interacting with $\phi_{0}$ background field. That is,

$$
\left.Z_{\text {String }}[\phi]\right|_{\phi \rightarrow \phi_{0}}=Z_{\mathrm{CFT}}\left[\phi_{0}\right] .
$$

In the large- $N$ case, the type IIB string correction to supergravity is proportional to $1 / \sqrt{g_{s} N}$, $g_{s}$ being the string coupling, thus one can neglect the string effect and just consider its lowenergy effective theory, the type IIB supergravity. In this case, the partition function of the type IIB superstring can be evaluated as the exponential of the supergravity action in a field configuration $\phi^{\mathrm{cl}}\left[\phi^{0}\right]$ which satisfies the classical equation of motion of the supergravity with the boundary condition given by $\phi_{0}$, i.e.,

$$
\left.Z_{\text {String }}[\phi]\right|_{\phi \rightarrow \phi_{0}}=\exp \left(-S_{\text {SUGRA }}\left[\phi^{\mathrm{cl}}\left[\phi_{0}\right]\right]\right) \text {. }
$$

Comparing (4) with (2) and (3), we immediately conclude that the background effective action of the large- $N$ limit of the $d$-dimensional conformal field theory can be approximately equal to the on-shell classical action of $A d S_{d+1}$ supergravity with non-empty boundary,

$$
\Gamma_{\mathrm{CFT}}\left[\phi_{0}\right]=S_{\mathrm{SUGRA}}\left[\phi^{\mathrm{cl}}\left[\phi_{0}\right]\right]=\int d^{d} x \sum_{n} \phi_{0}^{(n)}(x)\left\langle\mathcal{O}^{(n)}\right\rangle .
$$

In the above equation, $\mathcal{O}^{(r)}$ are the various composite operators in the superconformal field theory such as the energy-momentum tensor and chiral $R$-symmetry current etc. and $\phi_{0}^{(r)}$ 
are the corresponding background fields such as the gravitational and gauge fields etc., which are boundary values of the corresponding bulk fields.

Let us emphasize the role of the five-dimensional gauged supergravities [4-6] in AdS/CFT correspondence [7]. The $A d S_{5} \times S^{5}$ background arises from the near horizon limit of D3brane solution of type IIB supergravity [8]. In the $A d S_{5} \times S^{5}$ background, the spontaneous compactification on $S^{5}$ of the type IIB supergravity actually occurs $[9,10]$. With the assumption that there exists a consistent nonlinear truncation of the massless modes from the whole Kluza-Klein spectrum of the type IIB supergravity compactified on $S^{5}$ [10-12], the resultant theory should be the $S O(6)(\cong S U(4))$ gauged $\mathcal{N}=8 A d S_{5}$ supergravity since the isometry group $S O(6)$ of the internal space $S^{5}$ becomes the gauge group of the compactified theory and the $A d S_{5} \times S^{5}$ background preserves all of the supersymmetries of type IIB supergravity [6]. Furthermore, if the background for the type IIB supergravity is $A d S_{5} \times X^{5}$ with $X^{5}$ being an Einstein manifold rather than $S^{5}$ such as $T^{1,1}=(S U(2) \times S U(2)) / U(1)$ or certain orbifold, then due to the singularities in the internal manifold, the number of preserved supersymmetries in the compactified $A d S_{5}$ supergravity is reduced and the isometry group of the theory also changes [13-16]. One can thus obtain the gauged $\mathcal{N}=2,4 \operatorname{Ad} S_{5}$ supergravities in five dimensions, and their dual field theories are believed to be $N=1,2$ supersymmetric gauge theories $[7,14-16]$. In a strict sense, a supersymmetric gauge theory with lower supersymmetries is not a conformal invariant theory since its beta function does not vanish. However, it was shown that renormalization group flow of this type of supersymmetric gauge theory has the fixed point, at which the conformal invariance can arise [15-21]. The AdS/CFT correspondence between the $\mathcal{N}=2,4$ gauged supergravities in five dimensions and $\mathcal{N}=1,2$ supersymmertric gauge theories can thus be established [7,14-16].

With the truncation from the Kaluza-Klein tower, Eq. (5) can be considered as a quantum effective action describing a superconformal gauge theory in an external supergravity background [3,22], only where the external fields are provided by the boundary values of those in a one-dimension-higher bulk space-time. It is well known that the superconformal anomaly will arise from a classical superconformal gauge theory in an external supergravity background. The reproduction of the superconformal anomaly from the bulk gauged supergravity will provide an important support to the above $A d S / C F T$ correspondence conjecture at low-energy level.

In the next section, we shall briefly introduce the superconformal anomaly for the $\mathcal{N}=1$ supersymmetric gauge theory in an external $\mathcal{N}=1$ conformal supergravity background. Section 3 is devoted to a review of the $\mathcal{N}=2$ gauged supergravity in five dimensions and its $A d S_{5}$ classical solution. We also emphasize the behaviour of the fields of gauged supergravity near the $A d S_{5}$ boundary and the reduction of bulk supersymmetry transformation into an $\mathcal{N}=1$ superconformal transformation on the $A d S_{5}$ boundary. It is well known that the $\mathcal{N}=1$ superconformal transformation consists of the $\mathcal{N}=1$ four-dimensional supersymmetry transformation and the special conformal supersymmetry transformation (or super-Weyl in curved space-time background). In section 4, we calculate the supersymmetry variation of the five-dimensional $\mathcal{N}=2$ gauged supergravity and extract out the surface term. Furthermore, considering the fact that the four-dimensional supersymmetry and the super-Weyl symmetry cannot be preserved simultaneously, we find the external gauge field part of the holographic super-Weyl anomaly of $\mathcal{N}=1$ supersymmetric gauge theory from the boundary term the five-dimensional gauged supergravity. In Section 5 we summarize 
the main results and present some further discussions. Our results suggest that the contributions to the superconformal anomaly from the external vector field and gravitational field may have different holographic origin.

\section{SUPERCONFORMAL ANOMALY MULTIPLET IN EXTERNAL CONFORMAL SUPERGRAVITY BACKGROUND}

A general four-dimensional $\mathcal{N}=1$ supersymmetric $S U(N)$ gauge theory consists of the $\mathcal{N}=1$ supersymmetric Yang-Mills theory coupled with $\mathcal{N}=1$ massless matter fields in various possible representations of the gauge group. The classical Lagrangian density is

$$
\mathcal{L}=\operatorname{Tr}\left(-\frac{1}{4} W_{\mu \nu} W^{\mu \nu}+\frac{1}{2} i \bar{\lambda} \gamma^{\mu} \nabla_{\mu} \lambda\right)+\mathcal{L}_{\text {matter }}
$$

where $W_{\mu \nu}$ is the field strength for the $S U(N)$ gauge field and the $\lambda$ is a Majorana spinor in the adjoint representation of $S U(N)$. Due to the supersymmetry, its energy-momentum tensor $\theta^{\mu \nu}$, the supersymmetry current $s^{\mu}$ and the axial vector (or equivalently chiral) Rcurrent $j^{(5) \mu}$ lie in a supermultiplet [23,24]. These currents at classical level are not only conserved,

$$
\partial_{\mu} \theta^{\mu \nu}=\partial_{\mu} s^{\mu}=\partial_{\mu} j^{(5) \mu}=0,
$$

but also satisfy further algebraic constraints

$$
\theta_{\mu}^{\mu}=\gamma_{\mu} s^{\mu}=0
$$

This will promote the Poincaré supersymmetry to a superconformal symmetry since one can construct three more conserved currents,

$$
d^{\mu} \equiv x_{\nu} \theta^{\nu \mu}, k_{\mu \nu} \equiv 2 x_{\nu} x^{\rho} \theta_{\rho \mu}-x^{2} \theta_{\mu \nu}, l_{\mu} \equiv i x^{\nu} \gamma_{\nu} s_{\mu}
$$

These three new conserved currents give the generators for dilatation, conformal boost and special supersymmetry transformation. However, the superconformal symmetry may become anomalous at quantum level. In the case that all of them, the trace of energy-momentum tensor, $\theta_{\mu}^{\mu}$, the $\gamma$-trace of supersymmetry current, $\gamma^{\mu} s_{\mu}$ and the divergence of the chiral $R$-current, $\partial_{\mu} j^{(5) \mu}$, get contribution from quantum effects,

$$
\left(\partial_{\mu} j^{(5) \mu}, \gamma^{\mu} s_{\mu}, \theta_{\mu}^{\mu}\right)
$$

will form a (on-shell) chiral supermultiplet with the $\partial_{\mu} j^{(5) \mu}$ playing the role of the lowest component of the corresponding composite chiral superfield [24-26].

In general, there are two possible sources for above chiral supermultiplet anomaly [26]. One is due to the non-vanishing beta function of $\mathcal{N}=1$ supersymmetric Yang-Mills theory; The other one comes from the coupling of above supercurrent multiplet with the external supergravity fields. In this paper, we shall concentrate on the superconformal anomaly arising from the latter one. In this case, the $\gamma$-trace anomaly consists only of the superWeyl anomaly since the corresponding special conformal supersymmetry transformation is 
just the supersymmetric analog of the Weyl (or local scale) transformation. Note that in a supersymmetric gauge theory, the Poincaré symmetry corresponding to the energymomentum tensor $\theta_{\mu \nu}$, the supersymmetry corresponding to the supersymmetry current $s_{\mu}$, and the chiral $R$-symmetry to the axial vector current $j_{\mu}^{(5)}$ are all global symmetries and there no gauge fields within the supersymmetric gauge theory itself to couple with them. If there are some external supergravity fields $g_{\mu \nu}$, axial vector fields $A_{\mu}$ and vector-spinor (Rarita-Schwinger) fields $\psi_{\mu}$ couple to $\theta^{\mu \nu}, j_{\mu}^{(5)}$ and $s_{\mu}$, respectively,

$$
\mathcal{L}_{\text {ext }}=\int d^{4} x \sqrt{-g}\left(g_{\mu \nu} \theta^{\mu \nu}+A_{\mu} j^{(5) \mu}+\bar{\psi}_{\mu} s^{\mu}\right),
$$

there will arise external superconformal anomaly chiral supermultiplet. The action (11) describing the coupling of the external supergravity fields with the currents of supersymmetric Yang-Mills theory shows that the covariant conservations of the currents, $\nabla_{\mu} \theta^{\mu \nu}=\nabla_{\mu} s^{\mu}=0$, are equivalent to the local gauge transformation invariance of the external supergravity system,

$$
\begin{aligned}
\delta g_{\mu \nu}(x) & =\nabla_{\mu} \xi_{\nu}+\nabla_{\nu} \xi_{\mu}, \\
\delta \psi_{\mu}(x) & =\nabla_{\mu} \chi(x) .
\end{aligned}
$$

Furthermore, the covariant conservation of the axial vector current $j_{\mu}^{(5)}$ and the vanishing of both the $\gamma$-trace of supersymmetry current and the trace of energy-momentum tensor at classical level,

$$
\nabla_{\mu} j^{(5) \mu}=\gamma^{\mu} s_{\mu}=\theta^{\mu}{ }_{\mu}=0,
$$

mean the Weyl transformation invariance of $g_{\mu \nu}$, the super-Weyl symmetry and the $U(1)$ chiral gauge symmetry of the corresponding external supergravity system,

$$
\begin{aligned}
\delta g_{\mu \nu} & =g_{\mu \nu} \sigma(x), \\
\delta \psi_{\mu} & =\gamma_{\mu} \eta(x), \\
\delta A_{\mu}(x) & =\partial_{\mu} \Lambda(x) .
\end{aligned}
$$

The transformations (12) and (14) imply that the external fields

$$
\left(g_{\mu \nu}, \psi_{\mu}, A_{\mu}\right)
$$

constitute an off-shell $\mathcal{N}=1$ conformal supergravity multiplet [27,28]. Therefore, in the context of the $A d S / C F T$ (or more generally gravity/gauge) correspondence the superconformal anomaly in $\mathcal{N}=1$ supersymmetric gauge theory due to the supergravity external sources will be reflected in the explicit violations of the bulk symmetries of $\mathcal{N}=2$ gauged $A d S_{5}$ supergravity on the boundary $[3,29,30]$.

With no consideration on the quantum correction from the dynamics of the supersymmetric gauge theory, the external superconformal anomaly is exhausted at one-loop level. The external superconformal anomaly multiplet for $\mathcal{N}=1$ pure supersymmetric Yang-Mills theory is listed as the following [26]: 


$$
\begin{aligned}
\nabla_{\mu} j^{(5) \mu} & =\frac{N^{2}-1}{16 \pi^{2}}\left(-\frac{1}{24} R_{\mu \nu \lambda \rho} \widetilde{R}^{\mu \nu \lambda \rho}+F_{\mu \nu} \widetilde{F}^{\mu \nu}\right) \\
\gamma_{\mu} s^{\mu} & =\frac{N^{2}-1}{16 \pi^{2}}\left(\frac{1}{16} R^{\mu \nu \lambda \rho} \gamma_{\lambda \rho}+\frac{1}{8} F_{\mu \nu}\right)\left(D_{\mu} \psi_{\nu}-D_{\nu} \psi_{\mu}\right), \\
\theta_{\mu}^{\mu} & =\frac{N^{2}-1}{16 \pi^{2}}\left(\frac{1}{8} C_{\mu \nu \lambda \rho} C^{\mu \nu \lambda \rho}-\frac{3}{16} \widetilde{R}_{\mu \nu \lambda \rho} \widetilde{R}^{\mu \nu \lambda \rho}+\frac{1}{3} F_{\mu \nu} F^{\mu \nu}\right) .
\end{aligned}
$$

In above equations, $\gamma_{\mu \nu}=i / 2\left[\gamma_{\mu}, \gamma_{\nu}\right] ; F_{\mu \nu}=\partial_{\mu} A_{\nu}-\partial_{\nu} A_{\mu}$ is the field strength corresponding to the external $U_{R}(1)$ vector field $A_{\mu} ; R_{\mu \nu \lambda \rho}$ and $C_{\mu \nu \lambda \rho}$ are the Riemannian and Weyl tensors corresponding to the gravitational background field $g_{\mu \nu} ; D_{\mu}$ is the covariant derivative with respect to both the external gravitational and gauge fields. The factor $N^{2}-1$ comes from the fact that the gauginoes are in the adjoint representation of $S U(N)$ gauge group and hence there are $N^{2}-1$ copies. Note that Eq. (16) contains only the contribution to the superconformal anomaly coming from the vector multiplet of $\mathcal{N}=1$ supersymmetric $S U(N)$ Yang-Mills theory. Naturally, there will also arise the contribution from the $\mathcal{N}=1$ chiral supermutiplets if they are present in the theory. Their contributions to the anomaly coefficient will be proportional to $N$ or $N^{2}-1$. This depends on the chiral multiplets belonging to the fundamental or adjoint representations of $S U(N)$ group.

In the context of the AdS/CFT correspondence, the holographical arising of the chiral $U_{R}(1)$ anomaly was pointed out by Witten that it should come directly from the ChernSimons term in the five-dimensional gauged supergravity [3]. Further, the holographic Weyl anomaly was shown in Ref. [29] through a procedure called holographic renormalization and in Ref. [31] by the holomorphic dimensional regularization and a special bulk diffeomorphism preserving the Fefferman-Graham metric [32] of an arbitrary $d+1$-dimensional manifold with boundary topologically isomorphic to $S^{d}$, respectively. Actually, there have arisen quite a number of papers to discuss various aspects of the holographic Weyl anomaly including the modified models [33], the asymptotically $A d S$ space-time [34], the subleading order of $1 / N$ correction $[35,36]$ and new calculation framework such as the Hamilton-Jacobi equation [37] etc. The aim of this paper is to tackle the holographic origin of the supersymmetry current anomaly $\gamma_{\mu} s^{\mu}$.

\section{III. $\mathcal{N}=2$ GAUGED SUPERGRAVITY IN FIVE DIMENSIONS AND ITS $A D S_{5}$ BOUNDARY REDUCTION}

To show clearly how the holographic supersymmetry current anomaly arises, we shall review some typical features of the $\mathcal{N}=2$ gauged supergravity in five dimensions.

The ungauged $\mathcal{N}=2$ supergravity in five dimensions has the same structure as $\mathcal{N}=1$ eleven-dimensional supergravity [38]. It has a global $U S p(2) \cong S U(2)$ R-symmetry, and it contains a graviton $E_{M}^{A}$, two gravitini $\Psi_{M}^{i}$ and a vector field $\mathcal{A}_{M}[4,39,40], M, A=0, \cdots, 5$ are the Riemannian and local Lorentz indices, respectively, and $i=1,2$ the $S U(2)$ doublet indices. The gravitini are the $U S p(2) \cong S U(2)$ doublets and the symplectic Majorana spinors. The classical Lagrangian density takes a simple form [39],

$$
E^{-1} \widetilde{\mathcal{L}}=-\frac{1}{2} \mathcal{R}[\Omega(E)]-\frac{1}{2} \bar{\Psi}_{M}^{i} \Gamma^{M N P} \nabla_{N} \Psi_{P i}-\frac{1}{4} a_{00} \mathcal{F}_{M N} \mathcal{F}^{M N}
$$




$$
\begin{aligned}
& -\frac{3}{8} \sqrt{\frac{1}{6}} i h_{0}\left(\bar{\Psi}_{M}^{i} \Gamma^{M N P Q} \Psi_{N i} \mathcal{F}_{P Q}+2 \bar{\Psi}^{M i} \Psi_{i}^{N} F_{M N}\right) \\
& +\frac{C}{6 \sqrt{6}} E^{-1} \epsilon^{M N P Q R} \mathcal{F}_{M N} \mathcal{F}_{P Q} \mathcal{A}_{R}+\text { four-fermi terms }
\end{aligned}
$$

where the covariant derivative on the spinor field is defined with the spinor connection $\Omega_{M}^{A B}$,

$$
\nabla_{M} \Psi_{N}^{i}=\left(\partial_{M}+\frac{1}{4} \Omega_{M}^{A B} \Gamma_{A B}\right) \Psi_{N}^{i}
$$

The gauging of above supergravity is just turning the $U(1)$ subgroup of the global $S U(2)$ R-symmetry group into a local gauge group and straightforwardly considering the vector field as the $U(1)$ gauge field [4]. The space-time covariant derivative on the gravitini will be enlarged to include the $U(1)$ gauge covariant derivative,

$$
D_{M} \Psi_{N}^{i}=\nabla_{M} \Psi_{N}^{i}+g \mathcal{A}_{M} \delta^{i j} \Psi_{N j} .
$$

The gauged $\mathcal{N}=2$ supergravity action is

$$
E^{-1} \mathcal{L}=E^{-1} \widetilde{\mathcal{L}}+g^{2} P_{0}^{2}-\frac{i \sqrt{6}}{8} g \bar{\Psi}_{M}^{i} \Gamma^{M N} \Psi_{N}^{j} \delta_{i j} P_{0} .
$$

The gauged $\mathcal{N}=2$ supergravity has $A d S_{5}$ classical solution that preserves $\mathcal{N}=2$ supersymmetry with the cosmological constant proportional to $P_{0}$ [4]. To make the $A d S_{5}$ classical solution take the standard form,

$$
\begin{aligned}
d s^{2} & =\frac{l^{2}}{r^{2}}\left[g_{\mu \nu}(x, r) d x^{\mu} d x^{\nu}-(d r)^{2}\right], \\
\mathcal{A}_{M} & =\Psi_{M}=0,
\end{aligned}
$$

and the full $\mathcal{N}=2$ supersymmetry preserved in this background, one must choose the parameters in the Lagrangian (20) as the following ones [4,41]:

$$
g=\frac{3}{4}, \quad h_{0}=\frac{l}{2} \sqrt{\frac{3}{2}}, \quad h^{0}=\frac{1}{h_{0}}, \quad V_{0}=1, \quad P_{0}=2 h^{0} V_{0}=\frac{4}{l} \sqrt{\frac{2}{3}}, \quad a_{00}=\left(h_{0}\right)^{2}=\frac{3 l^{2}}{8} .
$$

Consequently, the Lagrangian density (20) up to the quadratic terms in spinor fields becomes

$$
\begin{aligned}
E^{-1} \mathcal{L}= & -\frac{1}{2} \mathcal{R}-\frac{1}{2} \bar{\Psi}_{M}^{i} \Gamma^{M N P} D_{N} \Psi_{P i}-\frac{3 l^{2}}{32} \mathcal{F}_{M N} \mathcal{F}^{M N}+\frac{C}{6 \sqrt{6}} E^{-1} \epsilon^{M N P Q R} \mathcal{F}_{M N} \mathcal{F}_{P Q} \mathcal{A}_{R} \\
& -\frac{3 i}{4 l} \bar{\Psi}_{M}^{i} \Gamma^{M N} \Psi^{N j} \delta_{i j}-\frac{3 i l}{32}\left(\bar{\Psi}_{M}^{i} \Gamma^{M N P Q} \Psi_{N i} \mathcal{F}_{P Q}+2 \bar{\Psi}^{M i} \Psi_{i}^{N} \mathcal{F}_{M N}\right)-\frac{6}{l^{2}}
\end{aligned}
$$

The supersymmetry transformations at the leading order in spinor fields read

$$
\begin{aligned}
\delta E_{M}^{A} & =\frac{1}{2} \overline{\mathcal{E}}^{i} \Gamma^{A} \Psi_{M i}, \\
\delta \Psi_{M}^{i} & =D_{M} \mathcal{E}^{i}+\frac{i l}{16}\left(\Gamma_{M}^{N P}-4 \delta_{M}^{N} \Gamma^{P}\right) \mathcal{F}_{N P} \mathcal{E}^{i}+\frac{i}{2 l} \Gamma_{M} \delta^{i j} \mathcal{E}_{j}, \\
\delta \mathcal{A}_{M} & =\frac{i}{l} \bar{\Psi}_{M}^{i} \mathcal{E}_{i} .
\end{aligned}
$$


To calculate the holographic superconformal anomaly, we need to expand the fields around the $A d S_{5}$ vacuum solution (21). Geometrically, this is actually a process of revealing the asymptotic behaviour of the bulk fields near the boundary of $A d S_{5}$ space-time. Correspondingly, the various bulk symmetries will be reduced to those on the boundary. For examples, the bulk diffeormorphism invariance of the bulk decomposes into the diffeomorphism symmetry on the boundary and the Weyl symmetry [31], and the the bulk supersymmetry converts into a superconformal symmetry for an off-shell conformal supergravity on the boundary $[41,42]$.

The procedure of reducing the bulk gauged supergravity to the off-shell conformal supergravity on the boundary is displayed in a series of works on $A d S_{3} / C F T_{2}, A d S_{6} / C F T_{5}$ and $A d S_{7} / C F T_{6}$ by Nishimura et al [42]. The key point is using the equations of motion of the bulk fields to find their radial coordinate dependence near the boundary of $A d S_{5}$ space. For the spinor field such as the gravitino, one should also show how a symplectic Majorana spinor in five dimensions reduces to the chiral spinor on the four-dimensional boundary. The reduction from the on-shell five-dimensional $\mathcal{N}=2$ gauged supergravity to $\mathcal{N}=1$ off-shell conformal supergravity in four dimensions was performed by Balasubramanian et al. [41], so we briefly review their result and then use it to derive the holographic super-Weyl anomaly of the supersymmetry current in $\mathcal{N}=1$ supersymmetric gauge theory.

As the first step, one should partially fix the local symmetries of bulk supergravity in the radial direction. According to the $A d S_{5}$ solution (21), one can choose [41,42]

$$
E_{\mu}{ }^{a}(x, r)=\frac{l}{r} e_{\mu}{ }^{a}(x)+\mathcal{O}(r), \quad E_{r}{ }^{a}=E_{\mu}^{\bar{r}}=0, \quad E_{r}{ }^{\bar{r}}=\frac{l}{r},
$$

and

$$
\Psi_{r}^{i}(x, r)=0, \quad \mathcal{A}_{r}(x, r)=0,
$$

to fix the Lorentz symmetry, the supersymmetry and the gauge symmetry in the $r$-direction, respectively. The gauge-fixing choice and the torsion-free condition $d E^{a}+\Omega^{a}{ }_{b} \wedge E^{b}=0$, further determine the $r$-dependence of the spin connections,

$$
\begin{aligned}
& \Omega^{a}{ }_{\bar{r}}(x, r)=-E^{a}(x, r)=-\frac{l}{r} e^{a}(x), \\
& \Omega_{b}^{a}(x, r)=\omega_{b}^{a}(x) .
\end{aligned}
$$

In above equations, $\mu, a=0, \cdots, 3$ are the Riemannian and local Lorentz indices on the boundary, respectively, and $\bar{r}$ is the Lorentz index in the radial direction. We use the lower case quantities to denote the boundary values of bulk fields, i.e., they are independent of the radial coordinate $r$.

The linearized equation of motion for the gauge field $\mathcal{A}_{\mu}$ near the boundary,

$$
E^{-1} \partial_{M}\left[G^{M N} \partial_{N} \mathcal{A}_{\mu}(x, r)\right]=0,
$$

implies that at the leading order in $r$ one can choose $\mathcal{A}_{\mu}(x, r)$ to be independent of $r$,

$$
\mathcal{A}_{\mu}(x, r)=A_{\mu}(x) .
$$


Furthermore, the linearized equation of motion for the gravitino is

$$
\Gamma^{M N P} D_{N} \Psi_{P i}+\frac{3 i}{2} \Gamma^{M N} \Psi_{N}^{j} \delta_{i j}=0
$$

The reduced spin connection (27) and gauge field (29) as well as the gauge choices (25) and (26) lead to the boundary reduction of the bulk covariant derivative,

$$
\begin{aligned}
D_{r} & =\partial_{r}, \quad D_{\mu}=\widetilde{D}_{\mu}(x)-\frac{1}{2 r} \gamma_{\mu} \gamma_{5}, \\
\widetilde{D}_{\mu}(x) & \equiv \nabla_{\mu}+\frac{1}{4} \omega_{\mu}^{a b} \gamma_{a b}+\frac{3}{4} A_{\mu},
\end{aligned}
$$

where the four-dimensional convention is defined as the following,

$$
\gamma_{a}=\Gamma_{a}, \quad \Gamma_{\mu}=E_{\mu}^{a} \Gamma_{a}=\frac{l}{r} \gamma_{\mu}, \quad \Gamma^{\mu}=\bar{E}_{a}^{\mu} \Gamma^{a}=\frac{r}{l} \gamma^{\mu}, \quad \gamma_{5}=\Gamma^{\bar{r}}=\Gamma_{\bar{r}}, \quad \gamma_{5}^{2}=1
$$

The linearized gravitino equation reduces to

$$
\gamma^{\mu \nu}\left(\partial_{r} \delta_{i j}-\frac{1}{r} \delta_{i j}-\frac{3}{2 r} \gamma_{5} \epsilon_{i j}\right) \psi_{\nu}^{j}(x, r)-\gamma^{\mu \nu \rho} \widetilde{D}_{\nu}(x) \gamma_{5} \psi_{\rho i}(x, r)=0,
$$

Diagonalizing the above equation by combining the two components of the symplectic Majorana spinor, $\Psi_{\mu} \equiv \Psi_{\mu 1}+i \Psi_{\mu 2}$, and making the chiral decomposition $\Psi_{\mu}^{R} \equiv \frac{1}{2}\left(1-\gamma_{5}\right) \Psi_{\mu}$, $\Psi_{\mu}^{L} \equiv \frac{1}{2}\left(1+\gamma_{5}\right) \Psi_{\mu}$, one can see that near the boundary $r \rightarrow 0$, the equation for the righthanded component reads [41]

$$
\left(\partial_{r}+\frac{1}{2 r}\right) \Psi_{\mu}^{R}=0
$$

and hence the radial dependence of $\Psi_{\mu}^{R}$ is

$$
\Psi_{\mu}^{R}=\left(\frac{2 l}{r}\right)^{1 / 2} \psi_{\mu}^{R}(x) .
$$

The left-handed component is not independent, and its radial coordinate dependent behaviour turns out to be [41]

$$
\begin{aligned}
\Psi_{\mu}^{L} & =(2 l \tau)^{1 / 2} \chi_{\mu}^{L}(x), \\
\chi_{\mu}^{L} & =\frac{1}{3} \gamma^{\nu}\left(\widetilde{D}_{\mu} \psi_{\nu}^{R}-\widetilde{D}_{\nu} \psi_{\mu}^{R}\right)-\frac{i}{12} \epsilon_{\mu \nu}^{\lambda \rho} \gamma_{5} \gamma^{\nu}\left(\widetilde{D}_{\lambda} \psi_{\rho}^{R}-\widetilde{D}_{\rho} \psi_{\lambda}^{R}\right) .
\end{aligned}
$$

Therefore, the $\mathcal{N}=2$ bulk supergravity multiplet $\left(E_{M}^{A}, \Psi_{M}^{i}, \mathcal{A}_{M}\right)$ reduces to the boundary field $\left(e_{\mu}{ }^{a}, \psi_{\mu}^{R}, A_{\mu}\right)$. It is actually the $8+8$ off-shell multiplet of $\mathcal{N}=1$ conformal supergravity since the bulk supersymmetry transformation (24) reduces to the one for $\mathcal{N}=1$ conformal supergravity [41].

The boundary reduction of the bulk supersymmetric transformation to that for the conformal supergravity is the following. First, as done for the bulk gravitino, one must redefine 
the bulk supersymmetric transformation parameter, $\mathcal{E}(x, r)=\mathcal{E}_{1}(x, r)+i \mathcal{E}_{2}(x, r)$ and decompose it as the chiral components. The radial coordinate dependence of $\mathcal{E}^{L, R}$ should be the same as as the bulk gravitino,

$$
\mathcal{E}^{R}(x, r)=\left(\frac{2 l}{r}\right)^{1 / 2} \epsilon^{R}(x), \quad \mathcal{E}^{L}(x, r)=(2 l r)^{1 / 2} \eta^{L}(x) .
$$

At the leading order in $r$, the bulk supersymmetric transformations reduces to [41]

$$
\begin{aligned}
\delta e_{\mu}^{a} & =-\frac{1}{2} \bar{\psi}_{\mu} \gamma^{a} \epsilon, \\
\delta \psi_{\mu} & =\widetilde{D}_{\mu} \epsilon-\gamma_{\mu} \eta=\nabla_{\mu} \epsilon-\frac{3 i}{4} A_{\mu} \gamma_{5} \epsilon-\gamma_{\mu} \eta, \\
\delta A_{\mu} & =i\left(\bar{\psi}_{\mu} \gamma_{5} \eta-\bar{\chi}_{\mu} \gamma_{5} \epsilon\right),
\end{aligned}
$$

where all the spinorial quantities, $\psi_{\mu}(x), \chi_{\mu}(x) \epsilon(x)$ and $\eta(x)$ are Majorana spinors constructed from their chiral components $\psi_{\mu}^{R}(x), \chi_{\mu}^{L}(x), \epsilon^{R}(x)$ and $\eta^{L}(x)$. Eq. (38) shows that the reduced bulk supersymmetric transformation is indeed the supersymmetric transformation for $\mathcal{N}=1$ conformal supergravity with $\epsilon$ and $\eta$ playing the roles of parameters for supersymmetry and special supersymmetry transformations, respectively $[27,28]$.

\section{HOLOGRAPHIC SUPER-WEYL ANOMALY OF SUPERSYMMETRY CURRENT}

The arising of the super-Weyl anomaly in $\mathcal{N}=1$ supersymmetric Yang-Mills theory from the $\mathcal{N}=2$ gauged $A d S_{5}$ supergravity lies in two aspects. On one hand, as a supersymmetric field theory, the supersymmetric variation of the Lagrangian (23) of the gauged supergravity is a total derivative. These total derivative terms cannot be naively ignored due to the existence of the boundary $A d S_{5}$. On the other hand, near the $A d S_{5}$ boundary, the bulk supersymmetric transformation decomposes into the supersymmetry transformation and the super-Weyl transformation on the boundary. If we require supersymmetry on the boundary to be preserved, the total derivative terms should yield the super-Weyl anomaly of the supersymmetry current via the AdS/CFT correspondence given by Eq. (5).

In the following, we shall calculate the supersymmetric variation of the gauged $\mathcal{N}=2$ five-dimensional supergravity (23) and extract out the total derivative terms. Then we shall reduce it to the $A d S_{5}$ boundary and give the holographic supersymmetry current anomaly. However, it should be emphasized that this surface term only yields the gauge field background part of the super-Weyl anomaly. One must employ a holographic renormalization procedure to reveal the gravitational part, just as how the holographic Weyl anomaly was found in Ref. [29].

First, the supersymmetric variation of the pure gravitational term and the cosmological term gives

$$
\begin{aligned}
\delta S_{\mathrm{GR}} & =\delta \int d^{5} x E\left(-\frac{1}{2} \mathcal{R}-\frac{6}{l^{2}}\right)=\delta \int d^{5} x E\left(-\frac{1}{2} \bar{E}_{A}^{M} \bar{E}_{B}^{N} \mathcal{R}_{M N}{ }^{A B}-\frac{6}{l^{2}}\right) \\
& =\int d^{5} x E\left[-\frac{1}{2} \overline{\mathcal{E}}^{i} \gamma_{A} \Psi_{i}^{M}\left(\mathcal{R}_{M}^{A}-\frac{1}{2} E_{M}^{A} \mathcal{R}-\frac{6}{l^{2}} E_{M}^{A}\right)-\nabla_{M}\left(\bar{E}_{A}^{M} \bar{E}_{B}^{N} \delta \Omega_{N}{ }^{A B}\right)\right],
\end{aligned}
$$


where $\bar{E}_{A}^{M}$ is the inverse of $E_{M}^{A}$.

The supersymmetry variation of the pure gauge field terms (including Chern-Simons term) yields

$$
\begin{aligned}
\delta S_{\mathrm{GA}}= & \int d^{5} x \delta\left[E\left(-\frac{3 l^{2}}{32}\right) \mathcal{F}_{M N} \mathcal{F}^{M N}+\frac{C}{6 \sqrt{6}} \epsilon^{M N P Q R} F_{M N} \mathcal{F}_{P Q} \mathcal{A}_{R}\right] \\
= & \int d^{5} x\left[-\frac{3 l^{2}}{32}\left(\delta E \mathcal{F}_{M N} \mathcal{F}^{M N}+4 \mathcal{F}^{M N} \nabla_{M} \delta \mathcal{A}_{N}\right)\right. \\
& \left.+\frac{C}{6 \sqrt{6}} \epsilon^{M N P Q R}\left(4 \nabla_{M} \delta \mathcal{A}_{N} \mathcal{F}_{P Q} \mathcal{A}_{R}+\mathcal{F}_{M N} \mathcal{F}_{P Q} \delta \mathcal{A}_{R}\right)\right] \\
= & \int d^{5} x\left\{E \left[-\frac{3 l^{2}}{64} \overline{\mathcal{E}}^{i} \Gamma^{M} \Psi_{M i} \mathcal{F}_{N P} \mathcal{F}^{N P}\right.\right. \\
& \left.+\frac{3 i l}{8} \nabla_{M}\left(F^{M N} \overline{\mathcal{E}}^{i} \Psi_{N i}\right)-\frac{3 i l}{8}\left(\nabla_{M} \mathcal{F}^{M N}\right) \overline{\mathcal{E}}^{i} \Psi_{N i}\right] \\
& \left.-\frac{i C}{\sqrt{6} l} \epsilon^{M N P Q R}\left[\frac{2}{3} \nabla_{M}\left(\mathcal{A}_{R} \mathcal{F}_{P Q} \overline{\mathcal{E}}^{i} \Psi_{N i}\right)+\frac{1}{2} \mathcal{F}_{M N} \mathcal{F}_{P Q} \overline{\mathcal{E}}^{i} \Psi_{R i}\right]\right\} .
\end{aligned}
$$

As for the supersymmtric variations of the terms concerning the gravitino, we start from the kinetic terms of the gravitino,

$$
\begin{aligned}
\delta S_{\mathrm{KT}}= & \delta \int d^{5} x\left[-\frac{1}{2} E \bar{\Psi}_{M}^{i} \bar{E}_{A}^{M} \bar{E}_{B}^{N} \bar{E}_{C}^{P} \Gamma^{A B C}\left(\nabla_{N} \Psi_{P i}-\frac{3}{4} \mathcal{A}_{N} \delta_{i j} \Psi_{P}^{j}\right)\right] \\
= & \int d^{5} x\left(-\frac{1}{2}\right)\left[(\delta E) \bar{\Psi}_{M}^{i} \Gamma^{M N P} D_{N} \Psi_{P i}+E\left(\delta \bar{\Psi}_{M}^{i}\right) \Gamma^{M N P} D_{N} \Psi_{P i}\right. \\
& +3 E\left(\delta \bar{E}_{A}^{M}\right) \bar{E}_{B}^{N} \bar{E}_{C}^{P} \bar{\Psi}_{M}^{i} \Gamma^{A B C} D_{N} \Psi_{P i} \\
& \left.+\bar{\Psi}_{M}^{i} \Gamma^{M N P}\left(\nabla_{N} \delta \Psi_{P i}-\frac{3}{4} \delta_{i j}\left(\delta \mathcal{A}_{N} \Psi_{P}^{j}+\mathcal{A}_{N} \delta \Psi_{P}^{j}\right)\right)\right] .
\end{aligned}
$$

Considering only the terms at most quadratic in the spinor quantities, we have

$$
\begin{aligned}
\delta S_{\mathrm{KT}}= & \int d^{5} x\left(-\frac{1}{2}\right) E\left\{\left[\nabla_{M} \overline{\mathcal{E}}^{i}+\frac{3}{4} \mathcal{A}_{\mu} \delta^{i j} \overline{\mathcal{E}}_{j}-\frac{i l}{16} \overline{\mathcal{E}}^{i}\left(\Gamma_{M}^{R S}+4 \delta_{M}^{R} \Gamma^{S}\right) F_{R S}-\frac{i}{2 l} \delta^{i j} \overline{\mathcal{E}}_{j} \Gamma_{M}\right]\right. \\
& \times \Gamma^{M N P}\left(\nabla_{N} \Psi_{P i}-\frac{3}{4} \mathcal{A}_{N} \delta_{i k} \Psi_{P}^{k}\right) \\
& +\bar{\Psi}_{M}^{i} \Gamma^{M N P} \nabla_{N}\left[\nabla_{P} \mathcal{E}_{i}-\frac{3}{4} \mathcal{A}_{P} \delta_{i j} \mathcal{E}^{j}+\frac{i l}{16}\left(\Gamma_{P}^{R S}-4 \delta_{P}^{R} \Gamma^{S}\right) \mathcal{E}_{i} \mathcal{F}_{R S}+\frac{i}{2 l} \Gamma_{P} \delta_{i k} \mathcal{E}^{k}\right] \\
& \left.-\frac{3}{4} \delta_{i j} \bar{\Psi}_{M}^{i} \Gamma^{M N P} \mathcal{A}_{N}\left[\nabla_{P} \mathcal{E}^{j}+\frac{3}{4} \mathcal{A}_{P} \delta^{j k} \mathcal{E}_{k}+\frac{i l}{16}\left(\Gamma_{P}^{R S}-4 \delta_{P}^{R} \Gamma^{S}\right) \mathcal{E}^{j} \mathcal{F}_{R S}-\frac{i}{2 l} \Gamma_{P} \delta^{j k} \mathcal{E}_{k}\right]\right\} \\
= & \int d^{5} x\left(-\frac{1}{2}\right) E\left\{\left[\left(\nabla_{M} \overline{\mathcal{E}}^{i}\right) \Gamma^{M N P} \nabla_{N} \Psi_{P i}+\bar{\Psi}_{M}^{i} \Gamma^{M N P} \nabla_{N} \nabla_{P} \mathcal{E}_{i}\right]\right. \\
& -\frac{3}{4}\left[\delta_{i j} \mathcal{A}_{N}\left(\nabla_{M} \overline{\mathcal{E}}^{i} \Gamma^{M N P} \Psi_{P}^{j}+\bar{\Psi}_{M}^{i} \Gamma^{M N P} \nabla_{P} \mathcal{E}^{j}\right)\right. \\
& \left.-\mathcal{A}_{M} \delta^{i j} \overline{\mathcal{E}}_{j} \Gamma^{M N P} \nabla_{M} \Psi_{P i}+\delta_{i j} \bar{\Psi}_{M}^{i} \Gamma^{M N P} \nabla_{N}\left(\mathcal{A}_{P} \mathcal{E}^{j}\right)\right](2)
\end{aligned}
$$




$$
\begin{aligned}
& +\frac{i l}{16}\left[-\mathcal{F}_{R S} \overline{\mathcal{E}}^{i} \Gamma_{M}^{R S} \Gamma^{M N P} \nabla_{N} \Psi_{P i}+\bar{\Psi}_{M}^{i} \Gamma^{M N P} \nabla_{N}\left(\Gamma_{P}^{R S} \mathcal{F}_{R S} \mathcal{E}_{i}\right)\right] \\
& +\frac{i l}{16} \frac{3}{4} \mathcal{A}_{N} \mathcal{F}_{R S} \delta_{i j}\left(\overline{\mathcal{E}}^{i} \Gamma_{M}^{R S} \Gamma^{M N P} \Psi_{P}^{j}-\bar{\Psi}_{M}^{i} \Gamma^{M N P} \Gamma_{P}^{R S} \mathcal{E}^{j}\right) \\
& -\frac{i l}{4}\left[\mathcal{F}_{M R} \overline{\mathcal{E}}^{i} \Gamma^{R} \Gamma^{M N P} \nabla_{N} \Psi_{P i}+\bar{\Psi}_{M}^{i} \Gamma^{M N P} \nabla_{N}\left(\Gamma^{R} F_{P R} \mathcal{E}_{i}\right)\right] \\
& +\frac{i l}{4} \frac{3}{4} \delta_{i j} \mathcal{A}_{N}\left(\mathcal{F}_{M R} \overline{\mathcal{E}}^{i} \Gamma^{R} \Gamma^{M N P} \Psi_{P}^{j}+\mathcal{F}_{P R} \bar{\Psi}_{M}^{i} \Gamma^{M N P} \Gamma^{R} \mathcal{E}^{j}\right) \\
& -\frac{i}{2 l}\left[\delta^{i j} \overline{\mathcal{E}}_{j} \Gamma_{\mu} \Gamma^{M N P} \nabla_{N} \Psi_{P i}-\delta_{i j} \bar{\Psi}_{M}^{i} \Gamma^{M N P} \nabla_{N}\left(\Gamma_{P} \mathcal{E}^{j}\right)\right] \\
& \left.+\frac{3}{4} \frac{i}{2 l} \mathcal{A}_{N} \delta^{i}{ }_{j}\left(\overline{\mathcal{E}}_{i} \Gamma_{M} \Gamma^{M N P} \Psi_{P}^{j}+\bar{\Psi}_{M}^{j} \Gamma^{M N P} \Gamma_{P} \mathcal{E}_{i}\right)\right\}
\end{aligned}
$$

We list the calculation results in the following:

$$
\begin{aligned}
(1)= & \nabla_{M}\left(\overline{\mathcal{E}}^{i} \Gamma^{M N P} \nabla_{N} \Psi_{P i}\right)-\overline{\mathcal{E}}^{i}\left[\nabla_{M}, \Gamma^{M N P}\right] \nabla_{N} \Psi_{P i} \\
& -\frac{1}{8} R_{M N A B} \overline{\mathcal{E}}^{i}\left(\Gamma^{M N P} \Gamma^{A B}+\Gamma^{A B} \Gamma^{M N P}\right) \Psi_{P i} \\
= & \nabla_{M}\left(\overline{\mathcal{E}}^{i} \Gamma^{M N P} \nabla_{N} \Psi_{P i}\right)+\left(R_{M}^{N}-\frac{1}{2} R \delta_{M}^{N}\right) \overline{\mathcal{E}}^{i} \Gamma^{M} \Psi_{N i} ; \\
\text { (2) }= & -\frac{3}{4} \nabla_{M}\left(\delta^{i j} \mathcal{A}_{N} \overline{\mathcal{E}}_{i} \Gamma^{M N P} \Psi_{P j}\right)+\frac{3}{4} \overline{\mathcal{E}}^{i} \Gamma^{M N P} \Psi_{M}^{j} \delta_{i j} \mathcal{F}_{N P} ; \\
\text { (3) }= & \frac{i l}{16} \nabla_{M}\left(\mathcal{F}_{R S} \overline{\mathcal{E}}^{i} \Gamma_{P}^{R S} \Gamma^{M N P} \Psi_{N i}\right)-\frac{i l}{8} \mathcal{F}_{R S} \overline{\mathcal{E}}^{i} \Gamma^{N P R S}\left(\nabla_{N} \Psi_{P}\right)_{i}+\frac{3 i l}{4} \mathcal{F}^{M N} \overline{\mathcal{E}}^{i}\left(\nabla_{M} \Psi_{N}\right)_{i} ; \\
\text { (4) }= & \frac{3 i l}{32} \mathcal{F}_{R S} \mathcal{A}_{M} \delta_{i j} \overline{\mathcal{E}}^{i} \Gamma^{M N R S} \Psi_{N}^{j}-\frac{9 i l}{16} \delta_{i j} \mathcal{F}^{M N} \mathcal{A}_{M} \overline{\mathcal{E}}^{i} \Psi_{N}^{j} ; \\
\text { (5) }= & \frac{i l}{4} \nabla_{M}\left(\overline{\mathcal{E}}^{i} \Gamma^{S} \Gamma^{M N P} \Psi_{N}^{i} \mathcal{F}_{P S}\right)+\frac{i l}{2} \mathcal{F}_{M R} \overline{\mathcal{E}}^{i} \Gamma^{M N P R}\left(\nabla_{N} \Psi_{P}\right)_{i} ; \\
\text { (6) }= & -\frac{3 i l}{8} \mathcal{F}_{M R} \mathcal{A}_{N} \delta_{i j} \overline{\mathcal{E}}^{i} \Gamma^{M N P R} \Psi_{P}^{j} ; \\
\text { (7) }= & \frac{i}{2 l} \delta_{i j}\left[-3 \overline{\mathcal{E}}^{i} \Gamma^{M N} \nabla_{M} \Psi_{N}^{j}+\bar{\Psi}_{M}^{i}\left[\Gamma^{M N P}, \nabla_{N}\right]\left(\Gamma_{P} \mathcal{E}^{j}\right)+3 \bar{\Psi}_{M}^{i} \nabla_{N}\left(\Gamma^{M N} \mathcal{E}^{j}\right)\right] \\
= & \frac{3 i}{2 l} \nabla_{M}\left(\overline{\mathcal{E}}^{i} \Gamma^{M N} \Psi_{N}^{j}\right)-\frac{3 i}{l} \delta^{i j} \overline{\mathcal{E}}^{i} \Gamma^{M N} \nabla_{M} \Psi_{N}^{j} ; \\
\text { (8) }= & -\frac{9 i}{4 l} \mathcal{A}_{M} \overline{\mathcal{E}}^{i} \Gamma^{M N} \Psi_{N i} .
\end{aligned}
$$

The supersymmetric variation of the gravitino mass term is

$$
\begin{aligned}
\delta S_{\mathrm{GM}}= & \delta\left[\frac{3 i}{4 l} \int E \bar{\Psi}_{M}^{i} \Gamma^{M N} \Psi_{N}^{j} \delta_{i j}\right]=\frac{3 i}{4 l} \int d^{5} x E\left[\left(\delta \bar{\Psi}_{M}^{i}\right) \Gamma^{M N} \Psi_{N}^{j}+\bar{\Psi}_{M}^{i} \Gamma^{M N} \delta \Psi_{N}^{j}\right] \delta_{i j} \\
= & \frac{3 i}{4 l} \int d^{5} x e\left\{\left[\nabla_{M} \overline{\mathcal{E}}^{a}+\frac{3}{4} \mathcal{A}_{M} \delta^{i k} \overline{\mathcal{E}}_{k}-\frac{i l}{16} \overline{\mathcal{E}}^{i}\left(\Gamma_{M}^{P Q}+4 \delta_{M}^{P} \Gamma^{Q}\right) F_{P Q}\right.\right. \\
& \left.-\frac{i}{2 l} \delta^{a c} \overline{\mathcal{E}}_{k} \Gamma_{M}\right] \Gamma^{M N} \Psi_{N}^{b} \\
& \left.+\bar{\Psi}_{M}^{i} \Gamma^{M N}\left[\nabla_{N} \mathcal{E}^{j}+\frac{3}{4} \mathcal{A}_{N} \delta^{j k} \mathcal{E}_{k}+\frac{i l}{16}\left(\Gamma_{N}-4 \delta_{N}^{P} \Gamma^{Q}\right) \mathcal{E}^{j} \mathcal{F}_{P Q}-\frac{i}{2 l} \Gamma_{N} \delta^{j k} \mathcal{E}_{k}\right]\right\} \delta_{i j}
\end{aligned}
$$




$$
\begin{aligned}
= & \frac{3 i}{4 l} \int d^{5} x E\left\{\delta_{i j}\left[\left(\nabla_{M} \overline{\mathcal{E}}^{i}\right) \Gamma^{M N} \Psi_{N}^{j}+\bar{\Psi}_{M}^{i} \Gamma^{M N} \nabla_{N} \mathcal{E}^{j}\right]\right. \\
& +\frac{3}{4} \delta^{i}{ }_{j}\left(\mathcal{A}_{M} \overline{\mathcal{E}}_{i} \Gamma^{M N} \Psi_{N}^{j}+\mathcal{A}_{N} \bar{\Psi}_{M}^{j} \Gamma^{M N} \mathcal{E}_{i}\right) \\
& -\frac{i l}{16} \delta_{i j} \mathcal{F}_{R P}\left(\overline{\mathcal{E}}^{i} \Gamma_{M}^{R P} \Gamma^{M N} \Psi_{N}^{j}-\bar{\Psi}_{M}^{i} \Gamma^{M N} \Gamma_{N}^{R P} \mathcal{E}^{j}\right) \\
& -\frac{i l}{4} \delta_{i j}\left(\overline{\mathcal{E}}^{i} \Gamma^{P} \Gamma^{M N} \Psi_{N}^{j} \mathcal{F}_{M P}+\bar{\Psi}_{M}^{i} \Gamma^{M N} \Gamma^{P} \mathcal{F}_{N P} \mathcal{E}^{j}\right) \\
& -\frac{2 i}{l} \delta^{i}{ }_{j}\left(\overline{\mathcal{E}}_{i} \Gamma^{M} \Psi_{M}^{j}+\bar{\Psi}_{M}^{j} \Gamma^{M} \mathcal{E}_{i}\right) \\
= & \int d^{5} x E\left[\frac{3 i}{2 l}\left(\nabla_{M} \overline{\mathcal{E}}^{i}\right) \Gamma^{M N} \Psi_{N}^{j} \delta_{i j}-\frac{9 i}{8} \mathcal{A}_{M} \overline{\mathcal{E}}^{i} \Gamma^{M N} \Psi_{N i}\right. \\
& \left.+\frac{3}{16} \delta_{i j} \mathcal{F}^{M N} \overline{\mathcal{E}}^{i} \Gamma_{M} \Psi_{N}^{j}-\frac{3}{16} \delta_{i j} \mathcal{F}_{M N} \overline{\mathcal{E}}^{i} \Gamma^{M N P} \Psi_{P}^{j}-\frac{3}{l^{2}} \overline{\mathcal{E}}^{i} \Gamma^{M} \Psi_{M i}\right] .
\end{aligned}
$$

Finally, the supersymmetric variation of the interaction terms between the gravitino $\Psi_{M}$ and the graviphoton $A_{M}$ gives

$$
\begin{aligned}
& \delta \int d^{5} x E\left(-\frac{3 i l}{32}\right)\left(\bar{\Psi}_{M}^{i} \Gamma^{M N P Q} \Psi_{N i} \mathcal{F}_{P Q}+2 \bar{\Psi}_{M}^{i} \Psi_{i}^{N} \mathcal{F}_{M N}\right) \\
= & -\frac{3 i l}{32} \int d^{5} x E\left\{\mathcal { F } _ { P Q } \left(\left[\nabla_{M} \overline{\mathcal{E}}^{i}+\frac{3}{4} \mathcal{A}_{M} \delta^{i k} \overline{\mathcal{E}}_{k}-\frac{i l}{16} \overline{\mathcal{E}}^{i}\left(\Gamma_{M}^{R S}+4 \delta_{M}^{R} \Gamma^{S}\right) \mathcal{F}_{R S}\right.\right.\right. \\
& \left.-\frac{i}{2 l} \delta^{i k} \overline{\mathcal{E}}_{k} \Gamma_{M}\right] \Gamma^{M N P Q} \Psi_{N i} \\
& \left.+\bar{\Psi}_{M}^{i} \Gamma^{M N P Q}\left[\nabla_{Q} \mathcal{E}_{i}-\frac{3}{4} \mathcal{A}_{Q} \delta_{i j} \mathcal{E}^{j}+\frac{i l}{16}\left(\Gamma_{Q}^{R S}-4 \delta_{Q}^{R} \Gamma^{S}\right) \mathcal{E}_{i} \mathcal{F}_{R S}+\frac{i}{2 l} \Gamma_{Q} \delta_{i k} \mathcal{E}^{k}\right]\right) \\
& +2 \mathcal{F}^{M N}\left(\left[\nabla_{M} \overline{\mathcal{E}}^{i}+\frac{3}{4} \mathcal{A}_{M} \delta^{i k} \overline{\mathcal{E}}_{k}-\frac{i l}{16} \overline{\mathcal{E}}^{i}\left(\Gamma_{M}^{R S}+4 \delta_{M}^{R} \Gamma^{S}\right) F_{R S}-\frac{i}{2 l} \delta^{i k} \overline{\mathcal{E}}_{k} \Gamma_{M}\right] \Psi_{N i}\right. \\
& \left.+\bar{\Psi}_{M}^{i}\left[\nabla_{N} \mathcal{E}_{i}-\frac{3}{4} \mathcal{A}_{N} \delta_{i j} \mathcal{E}^{j}+\frac{i l}{16}\left(\Gamma_{N}^{R S}-4 \delta_{N}^{R} \Gamma^{S}\right) \mathcal{E}_{i} \mathcal{F}_{R S}+\frac{i}{2 l} \Gamma_{N} \delta_{i k} \mathcal{E}^{k}\right]\right) \\
& +\cdots\} \\
= & -\frac{3 i l}{32} \int d^{5} x E\left\{2 \left[F^{M N}\left(\nabla_{M} \overline{\mathcal{E}}^{i} \Psi_{N i}+\bar{\Psi}_{M}^{i} \nabla_{N} \mathcal{E}_{i}\right)+\frac{3}{4} \delta^{i j} \mathcal{A}_{M} \mathcal{F}^{M N}\left(\overline{\mathcal{E}}_{i} \Psi_{N j}+\bar{\Psi}_{N i} \mathcal{E}_{j}\right)\right.\right. \\
& +\frac{i l}{16} \mathcal{F}^{M N} \mathcal{F}^{P Q}\left(-\overline{\mathcal{E}}^{i} \Gamma_{M P Q} \Psi_{N i}+\bar{\Psi}_{M}^{i} \Gamma_{N P Q} \mathcal{E}_{i}\right)-\frac{i l}{4} \mathcal{F}^{M N}\left(\overline{\mathcal{E}}^{i} \Gamma^{P} \Psi_{N i} \mathcal{F}_{M P}+\bar{\Psi}_{M}^{i} \Gamma^{P} \mathcal{E}_{i} \mathcal{F}_{N P}\right) \\
& \left.-\frac{i}{2 l} \mathcal{F}^{M N}\left(\delta^{i j} \overline{\mathcal{E}}_{i} \Gamma_{M} \Psi_{N j}-\delta_{i j} \bar{\Psi}_{M}^{i} \Gamma_{N} \mathcal{E}^{j}\right)\right] \\
& +\mathcal{F}_{P Q}\left[\left(\nabla_{M} \overline{\mathcal{E}}^{i} \Gamma^{M N P Q} \Psi_{N i}+\bar{\Psi}_{M}^{i} \Gamma^{M N P Q} \nabla_{N} \mathcal{E}_{i}\right)\right. \\
& +\frac{3}{4}\left(\mathcal{A}_{M} \delta^{i j} \overline{\mathcal{E}}_{i} \Gamma^{M N P Q} \Psi_{N j}-\mathcal{A}_{N} \delta_{i j} \bar{\Psi}_{M}^{i} \Gamma^{M N P Q} \mathcal{E}^{j}\right) \\
& -\frac{i l}{16} \mathcal{F}_{R S}\left(\overline{\mathcal{E}}^{i} \Gamma_{M}^{R S} \Gamma^{M N P Q} \Psi_{N i}-\bar{\Psi}_{M}^{i} \Gamma^{M N P Q} \Gamma_{N}^{R S} \mathcal{E}_{i}\right) \\
& -\frac{i l}{4}\left(\overline{\mathcal{E}}^{i} \Gamma^{R} \Gamma^{M N P Q} \Psi_{N i} \mathcal{F}_{M R}+\bar{\Psi}_{M}^{i} \Gamma^{M N P Q} \Gamma^{R} \mathcal{E}_{i} \mathcal{F}_{N R}\right) \\
&
\end{aligned}
$$




$$
\begin{aligned}
& \left.\left.-\frac{i}{2 l}\left(\delta^{i j} \overline{\mathcal{E}}_{i} \Gamma_{M} \Gamma^{M N P Q} \bar{\Psi}_{N j}-\delta_{i j} \bar{\Psi}_{M}^{i} \Gamma^{M N P Q} \Gamma_{N} \mathcal{E}^{j}\right)\right]\right\} \\
= & \int d^{5} x E\left[-\frac{3 i l}{8}\left(\nabla_{M} \overline{\mathcal{E}}\right)^{i} \Psi_{N i} \mathcal{F}^{M N}-\frac{9 i l}{32} \delta^{i j} \mathcal{A}_{M} \mathcal{F}^{M N} \overline{\mathcal{E}}_{i} \Psi_{N j}\right. \\
& -\frac{3 i l}{16}\left(\nabla_{M} \overline{\mathcal{E}}\right)^{i} \Gamma^{M N P Q} \Psi_{N i} \mathcal{F}_{P Q}-\frac{9 i l}{64} \mathcal{A}_{M} \mathcal{F}_{P Q} \delta^{i j} \overline{\mathcal{E}}_{i} \Gamma^{M N P Q} \Psi_{N j} \\
& +\frac{3 l^{2}}{64} \mathcal{F}^{M N} \mathcal{F}_{M N} \overline{\mathcal{E}}^{i} \Gamma^{P} \Psi_{P i}+\frac{3 l^{2}}{64} E^{-1} \epsilon^{M N P Q R} \mathcal{F}_{M N} \mathcal{F}_{P Q} \overline{\mathcal{E}}^{i} \Psi_{R i} \\
& +\frac{3 l^{2}}{32} \overline{\mathcal{E}}^{i} \Gamma^{Q} \Psi_{N a} \mathcal{F}^{N P} \mathcal{F}_{P Q}-\frac{3 l^{2}}{32} \overline{\mathcal{E}}^{i} \Gamma_{P} \Psi_{N i} \mathcal{F}^{M P} \mathcal{F}_{M N} \\
& \left.-\frac{3}{16} \delta^{i j} \overline{\mathcal{E}}_{i} \Gamma_{M} \Psi_{N j} \mathcal{F}^{M N}+\frac{3}{16} \delta^{i j} \overline{\mathcal{E}}_{i} \Gamma^{M N P} \Psi_{M j} \mathcal{F}_{N P}\right] .
\end{aligned}
$$

Putting the above supersymmetric variations (39) — (45) together, we get

$$
\begin{aligned}
\delta S= & \int d^{5} x E \nabla_{M}\left(-\frac{9 i l}{16} \overline{\mathcal{E}}^{i} \Psi_{N i} \mathcal{F}^{M N}-\frac{1}{2} \overline{\mathcal{E}}^{i} \Gamma^{M N P} \nabla_{N} \Psi_{P i}+\frac{3}{8} \overline{\mathcal{E}}^{i} \Gamma^{M N P} \Psi_{P}^{j} \delta_{i j} \mathcal{A}_{N}\right. \\
& \left.-\frac{3 i l}{32} \overline{\mathcal{E}}^{i} \Gamma^{M N P Q} \Psi_{N i} \mathcal{F}_{P Q}+\frac{9}{4} \overline{\mathcal{E}}^{i} \Gamma^{M N} \Psi_{N}^{j} \delta_{i j}+\frac{2 i C}{3 \sqrt{6} l} E^{-1} \epsilon^{M N P Q R} \overline{\mathcal{E}}^{i} \Psi_{R} \mathcal{A}_{N} \mathcal{F}_{P Q}\right) \\
& +\int d^{5} x\left(-\frac{i C}{2 \sqrt{6} l}+\frac{3 l^{2}}{64}\right) \epsilon^{M N P Q R} \overline{\mathcal{E}}^{i} \Psi_{R} \mathcal{F}_{M N} \mathcal{F}_{P Q} .
\end{aligned}
$$

In above calculation, we have made use of the following relations,

$$
\begin{aligned}
\Psi^{i}= & C^{-1} \Omega^{i j} \bar{\Psi}_{j}^{T}=C^{-1} \bar{\Psi}^{i T}, \quad \bar{\Psi}^{i}=-\Psi^{i T} C, \\
& \bar{\Psi}^{i} \Gamma_{M_{1} \cdots M_{n}} \Phi_{i}=-\Psi^{i T} C \Gamma_{M_{1} \cdots M_{n}} C^{-1} \bar{\Phi}_{i}^{T} \\
= & \left\{\begin{array}{l}
-\Psi^{i T} \Gamma_{M_{1} \cdots M_{n}} \bar{\Phi}_{i}^{T}=\bar{\Phi}_{i} \Gamma_{M_{1} \cdots M_{n}} \Psi^{i}=-\bar{\Phi}^{i} \Gamma_{M_{1} \cdots M_{n}} \Psi_{i}, \quad n=0,1,4,5, \\
\Psi^{i T} \Gamma_{M_{1} \cdots M_{n}} \bar{\Phi}_{i}^{T}=-\bar{\Phi}_{i} \Gamma_{M_{1} \cdots M_{n}} \Psi^{i}=\bar{\Phi}^{i} \Gamma_{M_{1} \cdots M_{n}} \Psi_{i}, \quad n=2,3,
\end{array}\right. \\
\Gamma_{M N}= & \frac{1}{2}\left[\Gamma_{M}, \Gamma_{N}\right], \quad \Gamma^{M N P}=-\frac{1}{2 !} E^{-1} \epsilon^{M N P Q R} \Gamma_{Q R}, \\
\Gamma^{M N P Q}= & E^{-1} \epsilon^{M N P Q R} \Gamma_{R}, \quad \Gamma_{M N P Q R}=E \epsilon_{M N P Q R} . \\
\Gamma_{M N} \Gamma_{P Q}= & E \epsilon_{M N P Q R} \Gamma^{R}-\left(G_{M P} G_{N Q}-G_{M Q} G_{N P}\right), \\
\Gamma_{M} \Gamma_{N P}= & \Gamma_{M N P}+G_{M N} \Gamma_{P}-G_{M P} \Gamma_{N}, \\
\Gamma^{M N P} \nabla_{N} \nabla_{P} \Psi_{i}= & \frac{1}{2} \Gamma^{M N P}\left[\nabla_{N}, \nabla_{P}\right] \Psi_{i}=\frac{1}{8} \Gamma^{M N P} \mathcal{R}_{N P A B} \Gamma^{A B} \Psi_{i} .
\end{aligned}
$$

Due to the nocommutativity between $\nabla_{M}$ and $\Gamma_{M_{1} \cdots M_{n}}$, we reiteratively use the following operations,

$$
\Gamma_{M_{1} \cdots M_{n}} \nabla_{M}(\cdots)=\left[\Gamma_{M_{1} \cdots M_{n}}, \nabla_{M}\right](\cdots)+\nabla_{M}\left[\Gamma_{M_{1} \cdots M_{n}}(\cdots)\right] .
$$

It is convenient to choose the inertial coordinate system, i.e. the Christoffel symbol $\Gamma_{N P}^{M}=0$. Consequently, the metricity condition leads to

$$
\partial_{M} E_{N}^{A}=0
$$


and hence the modified spin connection,

$$
\begin{aligned}
\Omega_{M A B}= & \frac{1}{2} \bar{E}_{A}^{N}\left(\partial_{M} E_{N B}-\partial_{N} E_{M B}\right)-\frac{1}{2} \bar{E}_{B}^{N}\left(\partial_{M} E_{N A}-\partial_{N} E_{M A}\right) \\
& -\frac{1}{2} \bar{E}_{A}^{P} \bar{E}_{B}^{Q}\left(\partial_{P} E_{Q c}-\partial_{Q} E_{P C}\right) E_{M}^{C}+\frac{1}{4}\left(\bar{\Psi}_{M} \Gamma_{A} \Psi_{B}+\bar{\Psi}_{A} \Gamma_{M} \Psi_{B}-\bar{\Psi}_{M} \Gamma_{B} \Psi_{A}\right),
\end{aligned}
$$

keeps only the quadratic fermionic terms. We have also considered the Ricci and Bianchi identities for the Riemannian curvature tensor and the Abelian gauge field

$$
\epsilon^{M N P Q R} \mathcal{R}_{S P Q R}=0, \quad \epsilon^{M N P Q R} \nabla_{N} \mathcal{R}_{S T P Q}=0, \quad \epsilon^{M N P Q R} \nabla_{N} \mathcal{F}_{Q R}=0 .
$$

If we choose the indefinite Chern-Simons coefficient in Eq. (46) as

$$
C=-\frac{3 i \sqrt{6} l^{3}}{32},
$$

the above supersymmetric variation of $\mathcal{N}=2$ gauged supergravity action in five dimensions is a total derivative,

$$
\begin{aligned}
\delta S & =\int d^{4} x \int_{r=\epsilon} d r \partial_{M}\left[E \left(-\frac{9 i l}{16} \overline{\mathcal{E}}^{i} \Psi_{N i} \mathcal{F}^{M N}-\frac{1}{2} \overline{\mathcal{E}}^{i} \Gamma^{M N P} \nabla_{N} \Psi_{P i}+\frac{3}{8} \overline{\mathcal{E}}^{i} \Gamma^{M N P} \Psi_{P}^{j} \delta_{i j} \mathcal{A}_{N}\right.\right. \\
& \left.\left.-\frac{3 i l}{32} E^{-1} \epsilon^{M N P Q R} \overline{\mathcal{E}}^{i} \Gamma_{R} \Psi_{N i} \mathcal{F}_{P Q}+\frac{9}{4} \overline{\mathcal{E}}^{i} \Gamma^{M N} \Psi_{N}^{i} \delta_{i j}+\frac{l^{2}}{16} E^{-1} \epsilon^{M N P Q R} \overline{\mathcal{E}}^{i} \Psi_{R} \mathcal{A}_{N} \mathcal{F}_{P Q}\right)\right]
\end{aligned}
$$

which is the typical feature of a supersymmetric field theory.

Now we can extract out the holographic super-Weyl anomaly from above total derivative terms as Witten [3] did in finding out the chiral $R$-symmetry anomaly. Considering the $r$-dependence of bulk fields and of the supersymmetry transformation parameter $\mathcal{E}^{i}$ given in (25), (26), (27), (29), (32), (35), (36) and (37), and taking the boundary limit $\epsilon \rightarrow 0$ after we integrate over the radial coordinate to the near boundary cut-off $r=\epsilon$, one can see that the non-vanishing contribution comes only from the term of the form $E^{-1} \epsilon^{M N P Q R} \overline{\mathcal{E}}^{i} \Gamma_{R} \Psi_{N i} \mathcal{F}_{P Q}$. Thus we obtain

$$
\delta S=\frac{3 i l^{3}}{32} \int d^{4} x \epsilon^{\mu \nu \lambda \rho} F_{\nu \lambda} \bar{\eta} \gamma_{\rho} \chi_{\mu} .
$$

In deriving Eq. (54) we have used the fact that the metric on the boundary should be the induced metric

$$
\widetilde{g}_{\mu \nu}(x, r)=\frac{l^{2}}{r^{2}} g_{\mu \nu}(x, r)
$$

rather than $g_{\mu \nu}(x, r)$ [30]. Eq. (54) will lead to the super-Weyl anomaly in terms of the AdS/CFT correspondence since it is proportional to the special supersymmetry transformation parameter $\eta$. Considering Eq. (36), we have

$$
\begin{aligned}
\delta S & =\int d^{4} x \bar{\eta} \gamma^{\mu} s_{\mu} \\
& =\frac{3 i l^{3}}{32} \int d^{4} x \epsilon^{\mu \nu \lambda \rho} F_{\nu \lambda} \bar{\eta} \gamma_{5} \gamma_{\rho} \gamma^{\alpha}\left[\frac{1}{3}\left(D_{\mu} \psi_{\alpha}-D_{\mu} \psi_{\alpha}\right)-\frac{i}{6} \epsilon_{\mu \alpha \sigma \delta} \gamma_{5} D^{\sigma} \psi_{\delta}\right] \\
& =-\frac{l^{3}}{16} \int d^{4} x\left[F^{\mu \nu} D_{\mu} \psi_{\nu}+\epsilon^{\mu \nu \lambda \rho} \gamma_{5} F_{\mu \nu} D_{\lambda} \psi_{\rho}+\frac{1}{2} \sigma^{\mu \nu} F_{\nu \lambda}\left(D_{\mu} \psi^{\lambda}-D^{\lambda} \psi_{\mu}\right)\right]
\end{aligned}
$$


where we have used the $\gamma$-matrix algebraic relations,

$$
\gamma^{\mu} \gamma^{\nu}=g^{\mu \nu}-i \gamma^{\mu \nu}, \quad \gamma_{5} \gamma^{\mu \nu}=\frac{i}{2} \epsilon^{\mu \nu \lambda \rho} \gamma_{\lambda \rho} .
$$

If we switch on the overall gravitational constant factor $-1 /\left(8 \pi G^{(5)}\right)$ on the classical Lagrangian (23), and consider the following relations among the $A d S_{5}$ radius $l$, string coupling $g_{s}$, the number $N$ of the $D 3$-branes, the five- and ten-dimensional gravitational constants connected by the compactification on $S^{5}$ of radius $l$,

$$
G^{(5)}=\frac{G^{(10)}}{\text { Volume }\left(S^{5}\right)}=\frac{G^{(10)}}{l^{5} \pi^{3}}, \quad G^{(10)}=8 \pi^{6} g_{s}^{2}, \quad l=\left(4 \pi N g_{s}\right)^{1 / 4}
$$

Eq. (54) yields the gauge field part of the holographic super-Weyl anomaly,

$$
\begin{aligned}
\gamma_{\mu} s^{\mu} & =\frac{1}{8 \pi G^{(5)}} \frac{l^{3}}{16}\left[F^{\mu \nu} D_{\mu} \psi_{\nu}+\epsilon^{\mu \nu \lambda \rho} \gamma_{5} F_{\mu \nu} D_{\lambda} \psi_{\rho}+\frac{1}{2} \sigma^{\mu \nu} F_{\nu \lambda}\left(D_{\mu} \psi^{\lambda}-D^{\lambda} \psi_{\mu}\right)\right] \\
& =\frac{N^{2}}{64 \pi^{2}}\left[F^{\mu \nu} D_{\mu} \psi_{\nu}+\epsilon^{\mu \nu \lambda \rho} \gamma_{5} F_{\mu \nu} D_{\lambda} \psi_{\rho}+\frac{1}{2} \sigma^{\mu \nu} F_{\nu \lambda}\left(D_{\mu} \psi^{\lambda}-D^{\lambda} \psi_{\mu}\right)\right] .
\end{aligned}
$$

This is the super-Weyl anomaly of supersymmetry current contributed from the external gauge field background at the leading order of large- $N$ expansion of $\mathcal{N}=1 S U(N)$ supersymmetric gauge theory.

\section{SUMMARY AND DISCUSSION}

We have investigated the super-Weyl anomaly of $\mathcal{N}=1$ supersymmetric Yang-Mills theory in the external $\mathcal{N}=1$ conformal supergravity background via the AdS/CFT correspondence. With the speculation that at low-energy the type IIB supergravity in $A d S_{5} \times X^{5}$ background should reduce to the gauged supergravity in five dimensions since such a background provides a spontaneous compactification on $X^{5}$, there should exists a holographic correspondence between $\mathcal{N}=2$ conformal supergravity in five dimensions and $\mathcal{N}=1$ supersymmetric Yang-Mills theory at the fixed point of its renormalization group flow. The fivedimensional $\mathcal{N}=2$ gauged supergravity has an $A d S_{5}$ classical solution which preserves the full supersymmetry. Around this $A d S_{5}$ vacuum configuration, the five-dimensional $\mathcal{N}=2$ on-shell gauged supergravity multiplet reduces to the $\mathcal{N}=1$ off-shell conformal supergravity mutiplet on the boundary of $A d S_{5}$ space. Correspondingly, the bulk $\mathcal{N}=2$ supersymmetry transformation converts into the $\mathcal{N}=1$ superconformal transformation in four dimensions, which consists of the supersymmetry transformation for $\mathcal{N}=1$ Poincaré supergravity and super-Weyl transformation. With these facts in mind, we calculate the supersymmetry variation of the $\mathcal{N}=2$ gauged supergravity in five dimensions and obtain the total derivative terms. Further, we reduce the total derivative terms to the boundary of $A d S_{5}$ space using the boundary reduction of the bulk fields. Considering the incompatibility of the $\mathcal{N}=1$ Poincaré supersymmetry and the super-Weyl symmetry, we extract out the super-Weyl anomaly of $\mathcal{N}=1$ supersymmetric gauge theory.

However, as shown in Eq. (59), we only reveal the contribution from the external gauged field when $\mathcal{N}=1$ supersymmetric gauge theory couples to $\mathcal{N}=1$ conformal supergravity 
background. As shown in Ref. [43], there usually should also has a contribution relevant to the external gravitational background,

$$
\gamma_{\mu} s^{\mu} \sim \frac{N^{2}}{128 \pi^{2}} R^{\mu \nu \lambda \rho} \gamma_{\mu \nu} D_{\lambda} \psi_{\rho}
$$

The reason for not having revealed the contribution of the gravitational background field is not yet fully clear. However, since the super-Weyl anomaly shares a supermultiplet with the Weyl anomaly and the chiral R-symmetry anomaly, we have the following two speculations based on the process of deriving the gravitational background parts in both the holographic Weyl and chiral anomalies $[35,36]$.

First, it is possible that this term cannot be revealed within the five-dimensional $\mathcal{N}=2$ gauged supergravity itself, and one must consider the supersymmetric action consisting of higher-order gravitational terms such as the Gauss-Bonnet term. As given in Ref. [26], for a general $\mathcal{N}=1$ supersymmetric gauge theory with $N_{v}$ vector and $N_{\chi}$ chiral multiplets in an external supergravity background, the chiral R-symmetry anomaly and the Weyl anomaly take the forms,

$$
\begin{aligned}
\nabla_{\mu} j^{(5) \mu} & =\frac{c-a}{24 \pi^{2}} R_{\mu \nu \lambda \rho} \widetilde{R}^{\mu \nu \lambda \rho}+\frac{5 a-3 c}{9 \pi^{2}} F_{\mu \nu} \widetilde{F}_{\mu \nu} \\
\theta^{\mu}{ }_{\mu} & =\frac{c}{16 \pi^{2}} C_{\mu \nu \lambda \rho} C^{\mu \nu \lambda \rho}-\frac{a}{16 \pi^{2}} \widetilde{R}_{\mu \nu \lambda \rho} \widetilde{R}^{\mu \nu \lambda \rho}+\frac{c}{6 \pi^{2}} F_{\mu \nu} F^{\mu \nu} .
\end{aligned}
$$

The coefficients are purely determined by the field contents. For a supersymmetric theory in the weak coupling limit, they are [26]

$$
c=\frac{1}{24}\left(3 N_{v}+N_{\chi}\right), \quad a=\frac{1}{48}\left(9 N_{v}+N_{\chi}\right) .
$$

In the pioneering work by Witten [3], the chiral $R$-symmetry anomaly of $\mathcal{N}=4 S U(N)$ SYM in the large- $N$ limit is perfectly reproduced from the Chern-Simons (CS) term in $\mathcal{N}=8$ five-dimensional $S O(6)$ gauged supergravity. The reason is that the field contents of $\mathcal{N}=4$ SYM can be considered as one $\mathcal{N}=1$ vector multiplet plus three chiral multiplets in the adjoint representation of $S U(N)$, and this leads to $c=a=1 / 4$ [36]. Thus there is no anomalous term relevant to the gravitational background field in the chiral anomaly and the CS term is fully responsible for the holographic origin of the chiral R-symmetry anomaly. This also explains the absence of the $R_{\mu \nu \lambda \rho} R^{\mu \nu \lambda \rho}$ term in the holographic Weyl anomaly of $\mathcal{N}=4 \mathrm{SYM}$ found in Ref. [29]. For the $\mathcal{N}=1,2$ supersymmetric gauge theories, Eq. (62) shows that in general $a \neq c$. Thus the terms relevant to gravitational background in the superconformal anomaly should arise from the supergravity side. In Ref. [35], it was shown that for the $\mathcal{N}=2$ supersymmetric $U S p(2 N)$ gauge theory coupled to two hypermultiplets in the fundamental and antisymmetric tensor representations of the gauge group, respectively, the gravitational background part in the holographic chiral anomaly does originate from a mixed CS term. However, this CS terms is obtained from the compactification on $S^{3}$ of the Wess-Zumino term describing the interaction of the $\mathrm{R}$ - $\mathrm{R} 4$-form field with eight $D 7$-branes and one orientifold 7-plane system. Specifically, this gravitational background term is at the subleading $N$ order rather than the leading $N^{2}$ order in the large- $N$ expansion of $\mathcal{N}=2$ supersymmetric $U S p(2 N)$ gauge theory. Due to the supersymmetry, there should also arise 
the $N$-order part containing the $R_{\mu \nu \lambda \rho} R^{\mu \nu \lambda \rho}$ term in the holographic Weyl anomaly. This fact was explicitly shown in Ref. [36]. The authors first performed $T$-duality and realized that the above mixed CS term originates from Green-Schwarz couplings in the heterotic or type I supergravity. Further, they considered the higher-order gravitational terms required by the supersymmetry in the low-energy effective action of one-loop heterotic or type $I$ string theory [44] and then performed the heterotic-type $I$-type $I^{\prime}$ T-duality to extract the desired gravitational term in the $A d S_{5}$ supergravity. Finally, the holographic Weyl anomaly containing the square of the Riemannian tensor was found along the line of Ref. [29]. These two cases imply that we should consider the full supersymmetric action consisting of the higher-order gravitational terms and its supersymmetric variation in the $A d S_{5}$ background will most probably lead to the term (60) in the holographic super-Weyl anomaly. However, the brane configuration for the $\mathcal{N}=1 U(N)$ gauge theory is quite complicated, being a $D 4-D 6-N S 5-N S 5^{\prime}$ system [45]. It is not straightforward to write down the mixed CS term from the corresponding Wess-Zumino term and to extract the explicit form of the supersymmetric action containing the higher-order gravitational terms. We hope to make a complete investigation along the line similar to the one adopted in Refs. [35,36].

The other possible reason for the failure of getting the gravitational background contribution is that in Eqs.(25), (29), (35) and (36) we consider only the leading order of $r$-dependence of the bulk fields near the $A d S_{5}$ boundary. This argument is inspired by the process of deriving the holographic Weyl anomaly. It was shown in Ref. [29] that if one makes a complete near-boundary analysis and considers the asymptotic expansion beyond the leading order up to logarithmic term $[29,30]$, i.e.,

$$
\mathcal{F}(x, r)=r^{m}\left[f_{(0)}(x)+f_{(2)}(x) r+\cdots r^{n}\left(f_{(2 n)}(x)+\widetilde{f}_{(2 n)}(x) \ln r+\cdots\right)+\cdots\right],
$$

the higher-order gravitational terms can arise in the on-shell action [29], and they lead to the holographic Weyl anomaly composed of the $R_{\mu \nu} R^{\mu \nu}$ and $R^{2}$ terms. Therefore, it is also possible that the gravitational background field part in the super-Weyl anomaly can arise as the holographic Weyl anomaly does [29] if one takes into account the logarithmic terms in above expansion. Of course, in this case the on-shell gauged five-dimensional supergravity action has the infrared divergence near the $A d S_{5}$ boundary due to its infinite boundary. One must perform a holographic renormalization procedure to make it well defined [30]. However, although we do not exclude that this is a possible source, it seems that the first reasoning is a more convincing one.

We are not aware whether there exist any physical reasons for the difference between these two holographic contributions to the superconformal anomaly. Since the essence of the holographic anomaly is the anomaly inflow from the bulk theory $[46],{ }^{1}$ this is the reason why one can extract out the anomaly from a higher-dimensional bulk theory, Thus it might be relevant to the difference between the anomaly inflows contributed by the gravitational and gauge field backgrounds.

Finally, it is worth to emphasize another way of calculating the holographic Weyl anomaly proposed in Ref. [31]. This approach is independent of the concrete form of the classical

\footnotetext{
${ }^{1}$ We thank A. Kobakhidze for discussion on this point.
} 
bulk gravitational action, and depends purely on a special bulk diffeomorphism (called the "PBH" transformation [47]), which keeps the form of the Fefferman-Graham metric [32] of an arbitrary $d+1$-dimensional manifold with the boundary topologically isomorphic to $S^{d}$ invariant and reduces to a Weyl transformation on the boundary. With a choice on the holomorphic dimensional regularization, there emerges no logarithmic function of the radial coordinate in the near-boundary asymptotic expansion. Further, the invariance of a general bulk gravitational action, which admits an $A d S_{d+1}$ classical solution, under this particular diffeomorphism yields a Wess-Zumino-like consistency condition satisfied by the generating functional for the Weyl anomaly on the boundary ( $d$ being an even integer). Hence the hologrpahic Weyl anomaly can be extracted out and no holographic renormalization procedure is necessary [31]. The advantage of this approach is that one can avoid the complicated near-boundary analysis and the subsequent holographic renormalization procedure. One may consider to use this approach to calculate the super-Weyl anomaly of a supersymmetry current. However, there is one crucial obstacle to overcome. That is, one needs to find a supersymmetric generalization of above special bulk diffeomorphism, which should keep both the supersymmetry in the bulk and on the boundary. In such an approach, one must employ the holographic dimensional regularization to prevent the logarithmic dependence from emerging in the near-boundary asymptotic expansion. However, the dimensional regularization in general does not preserve the supersymmetry. This fact has cast a shadow on the application of the above approach to the evaluation of the holographic supercurrent anomaly.

\section{ACKNOWLEDGMENTS}

We are indebted to M. Grisaru, A. Kobakhidze, M. Nishimura and A. Schwimmer for discussions. We also would like to thank G. Kunstatter, R.G. Leigh, C. Montonen and M. Sheikh-Jabbari for useful remarks. This work is supported by the Academy of Finland under the Project No. 163394. 


\section{REFERENCES}

[1] J. Maldacena, Adv. Theor. Math. Phys. 2 (1998) 231.

[2] S.S. Gubser, I.R. Klebanov and A.M. Polyakov, Phys. Lett. B428 (1998) 105.

[3] E. Witten, Adv. Theor. Math. Phys. 2 (1998) 253.

[4] M. Günaydin, G. Sierra and P.K. Townsend, Nucl. Phys. B242 (1984) 244; Nucl. Phys. B253 (1985) 573.

[5] M. Awada and P.K. Townsend, Nucl. Phys. 255 (1985) 617; L.J. Romans, Nucl. Phys. B267 (1986) 433.

[6] M. Günaydin, L. J. Romans and N.P. Warner, Phys. Lett. B154 (1985) 268; Nucl. Phys. 272 (1986) 598.

[7] S. Ferrara, C. Fronsdal and A. Zaffaroni, Nucl. Phys. B532 (1998) 153; S. Ferrara and A. Zaffaroni, Phys. Lett. B431 (1998) 49.

[8] For a review, see O. Aharony, S.S. Gubser, J. Maldacena, H. Ooguri and Y. Oz, Phys. Rept. 323 (2000) 183.

[9] P.G.O. Freund and M.A. Rubin, Phys. Lett. B97 (1980) 233.

[10] For a review, see M. Duff, B.E.W. Nilsson and C.N. Pope, Phys. Rep. 130 (1986) 1.

[11] M. Günaydin and N. Marcus, Class. Quant. Grav. 2 (1985) L11.

[12] H.J. Kim, L.J. Romans and P. van Nieuwenhuizen, Phys. Rev. D32 (1985) 389.

[13] L.J. Romans, Phys. Lett. B153 (1984) 401.

[14] I.R. Klebanov and E. Witten, Nucl. Phys. B536 (1998) 536.

[15] S. Kachru and E. Silverstein, Phys. Rev. Lett. 80 (1998) 4855.

[16] A. Lawrence, N. Nekrasov and C. Vafa, Nucl. Phys. B533 (1998) 199.

[17] N.A. Novikov, M.A. Shifman, A.I. Vainshtein and V.I. Zakharov, Nucl. Phys. B229 (1983) 381.

[18] N. Seiberg, Nucl. Phys. B435 (1995) 129.

[19] R.G. Leigh and M.J. Strassler, Nucl. Phys. B447 (1995) 95.

[20] For earlier works discussing on the $\mathcal{N}=1$ superconformal symmetry based on the perturbative $\beta$-function, see P. West, Phys. Lett. B137 (1984) 371; A. Parkes and P. West, Phys. Lett. B138 (1984) 99; D.R.T. Jones and L. Mezincescu, Phys. Lett. B138 (1984) 293; S. Hamidi, J. Patera and J. Schwarz, Phys. Lett. B141 (1984) 349; W. Lucha and H. Neufeld, Phys. Lett. B174 (1986) 186; P. Piguet, K. Sibold, Phys. Lett. B177 (1986) 373; Int. J. Mod. Phys. A1 (1986) 913; Phys. Lett. B201 (1988) 241; C. Lucchesi, O. Piguet and K. Sibold, Helv. Phys. Acta, 61 (1988) 321. D.R. T. Jones, Nucl. Phys. B277 (1986) 153; A.V. Ermushev, D.I. Kazakov and O.V. Tarasov, Nucl. Phys. B281 (1987) 72; X.D. Jiang and X.J. Zhou, Phys. Lett. B197 (1987) 156; Phys. Lett. B216 (1989) 160; Phys. Rev. D42 (1990) 2109.

[21] For earlier works discussing on the $\mathcal{N}=2$ superconformal symmetry based on the perturbative $\beta$-function, see P. Howe, K. Stelle and P. West, Phys. Lett. B124 (1983) 55; I.G. Koh and S. Rajpoot, Phys. Lett. B135 (1984) 397; F.X. Dong, T.S. Tu, P.Y. Xue and X.J. Zhou, Phys. Lett. B140 (1984) 333; J.P. Derendinger, S. Ferrara and A. Masiero, Phys. Lett. B143 (1984) 133.

[22] H. Liu and A.A. Tseytlin, Nucl. Phys. B533 (1998) 88.

[23] J. Iliopoulos and B. Zumino, Nucl. Phys. B76 (1974) 310; S. Ferrara and B. Zumino, Nucl. Phys. B87 (1975) 207; P. Howe, K.S. Stelle and P.K. Townsend, Nucl. Phys. B192 (1981) 332. 
[24] For reviews, see M.F. Sohnius, Phys. Rep. 128 (1985) 39; P. West, Introduction to Supersymmertry and Supergravity (World Scientific, Singapore, 1990); M. Chaichian, W.F. Chen and C. Montonen, Phys. Rep. 346 (2001) 89.

[25] T.E. Clark, O. Piguet and K. Sibold, Nucl. Phys. B143 (1978) 445; O. Piguet and K. Sibold, Nucl. Phys. B196 (1982) 428; ibid (1982) 447.

[26] For a brief review, see M.T. Grisaru, Anomalies in Supersymmetric Theories, Invited talk given at NATO Advanced Study Institute on Gravitation: Recent Developments, Cargese, France, Jul 10-29, 1978; D. Anselmi, D.Z. Freedman, M.T. Grisaru and A.A. Johansen, Nucl. Phys. B526 (1998) 543.

[27] S. Ferrara, M. Kaku, P.K. Townsend, P. van Nieuwenhuizen, Nucl. Phys. B129 (1977) 125; M. Kaku, P.K. Townsend, P. van Nieuwenhuizen, Phys. Rev. D17 (1978) 3179; Phys. Lett. B69 (1977) 304; J. Crispim-Romao, Nucl. Phys. B145 (1978) 535; T. Kugo and S. Uehara, Nucl. Phys. B226 (1983) 49.

[28] For a review, see E.S. Fradkin and A. A. Tseytlin, Phys. Rep. 119 (1985) 233.

[29] M. Henningson and K. Skenderis, JHEP 9807 (1998) 023.

[30] M. Bianchi, D.Z. Freedman and K. Skenderis, Nucl. Phys. B631 (2002) 159; JHEP: 0108 (2001) 041.

[31] C. Imbimbo, A. Schwimmer, S. Theisen and S. Yankielowicz, Class. Quant. Grav. 17 (2000) 1129.

[32] C. Fefferman and C.R. Graham, "Conformal Invariants" in Elie Cartan et les Mathématiques d'aujourd'hui (Astérisque, 1985) P. 95.

[33] S. Nojiri and S.D. Odintsov, Phys. Lett. B444 (1998) 92; Int. J. Mod. Phys. A15 (2000) 413; Mod. Phys. Lett. A15 (2000) 1043; S. Nojiri, S.D. Odintsov, S. Ogushi, A. Sugamoto and M. Yamamoto, Phys. Lett. B465 (1999) 128.

[34] V. Balasubranian and P. Kraus, Comm. Math. Phys. 208 (1999) 3731; A.M. Awad and C.V. Johnson, Phys. Rev. D61 (2000) 084025.

[35] O. Aharony, J. Pawelczyk, S. Theisen and S. Yankielowicz, Phys. Rev. D60 (1999) 066001; P. Mansfield and D. Nolland, Phys. Lett. B495 (2000) 435; S.G. Naculich, H.J. Schnitzer and N. Wyllard, Int. J. Mod. Phys. A17 (2002) 2567.

[36] M. Blau, K.S. Narain and E. Gava, JHEP 9909 (1999) 018.

[37] J. Kalkkinen and D. Martelli, Nucl. Phys. B596 (2001) 215; J. Kalkkinen, D. Martelli and W. Mück, JHEP 0104 (2001) 036; M. Fukuma, S. Matsuura and T. Sakai, Prog. Theor. Phys. 104 (2000) 1089.

[38] E. Cremmer, B. Julia and J. Scherk, Phys. Lett. B76 (1978) 409.

[39] E. Cremmer, in Superspace and Supergravity, ed. by S.W. Hawking and M. Rocěk (Cambridge University Press, 1981).

[40] For a recent discussion relevant to five-dimensional $N=2$ gauged supergravity, see M. Günaydin and M. Zagermann, Nucl. Phys. B572 (2000) 131; Phys. Rev. D62 (2000) 044028; ibid D63 (2001) 064023. A. Ceresole and G. Dall'Agata, Nucl. Phys. B585 (2000) 143.

[41] V. Balasubramanian, E. Gimon, D. Minic and J. Rahmfeld, Phys. Rev. D63 (2001) 104009.

[42] M. Nishimura and Y. Tanii, Phys. Lett. B446 (1999) 37; Int. J. Mod. Phys. A14 (1999) 3731; Mod. Phys. Lett. A14 (1999) 2709. M. Nishimura, Nucl. Phys. B588 (2000) 471.

[43] L.F. Abbott, M.T. Grisaru and H.J. Schnitzer, Phys. Lett. B73 (1978) 71. 
[44] C.G. Callan, D. Friedan, E.J. Martinec and M.J. Perry, Nucl. Phys. B262 (1985) 293; L.J. Romans and N.P. Warner, Nucl. Phys. B273 (1986) 320; E. Bergshoeff, A. Salam and E. Sezgin, Nucl. Phys. B279 (1987) 659; Y. Cai and C. Nunez, Nucl. Phys. B287 (1987) 279; D.J. Gross and J.H. Sloan, Nucl. Phys. B291 (1987) 41; C.M. Hull and P.K. Townsend, Nucl. Phys. B301 (1988) 197; E. Bergshoeff and M. de Roo, Nucl. Phys. B328 (1989) 439.

[45] S. Elizur, A. Giveon and D. Kutasov, Phys. Lett. B400 (1997) 269; S. Elizur, A. Giveon, D. Kutasov, E. Robinovici and A. Schwimmer, Nucl. Phys. B505 (1997) 202.

[46] C.G. Callan and J.A. Harvey, Nucl. Phys. B250 (1985) 427; S. Naculich, Nucl. Phys. B296 (1988) 837.

[47] R. Penrose and W. Rindler, Spinors and Spacetime, Chapter 9, Volume 2 (Cambridge University Press, 1986); J.D. Brown and M. Henneaux, Commun. Math. Phys. 104 (1986) 207. 